\title{
Tra postfemminismo e sperimentali- smo: Romana Petri e il calore del linguaggio
}

\section{Grazia Menechella}

Romana Petri, giovane scrittrice italiana, esordisce nel ' 90 con una raccolta di racconti: Il gambero blu e altri racconti; ed è un esordio alla grande perché con questo libro vince i premi Rapallo e Mondello. Nel '91 esce il suo primo romanzo, Il ritratto del disarmo, e nel ' 93 il secondo romanzo, Il baleniere delle montagne. ${ }^{1}$

Romana Petri appartiene alla generazione di Sandra Petrignani, Ippolita Avalli, Paola Capriolo, Valeria Viganò; generazione su cui è difficile e prematuro fare un discorso critico complessivo. Si può, però, tentare un profilo delle varie scrittrici ed io provvederò a quello della Petri analizzando le sue modalità di scrittura in $I l$ gambero blu e altri racconti e Il ritratto del disarmo.

Le scrittrici della nuova generazione sembrano concordare sul fatto che c'è una netta separazione con la generazione precedente da cui hanno "esplicitamente" preso le distanze; nelle parole della Petri:

La passione, talvolta anche donchisciottesca ma sempre nello spirito del tempo, che scrittrici come la Maraini - e molte altre - hanno espresso nelle loro opere, per noi non esiste: il nostro mondo è cinico e asettico. (Rusconi 99)

E Dacia Maraini così risponde:

Le donne che oggi scrivono quasi sempre partono dalla soggettività femminile: che importanza ha se non chiamano questo "femminismo"? (Rusconi 99)

Diversi i tempi e diverso il femminismo: siamo lontane dal femminismo storico/impegnato, lontane da racconti autobiografici e da storie di donne alla ricerca di sé. La Petri afferma:

I miei racconti non sono strettamente autobiografici, spesso nascono da un sogno, da una visione, da una proiezione fantastica .... (Pizzuto)

e la Maraini:

Young authors are at ease with third person narratives: they have a more objective view of literature, of reality; they have abandoned the realm of sheer autobiography that has shaped the writing of women so long. Some writers 
(Morazzoni, Capriolo, Mancinelli) opt for a particular fairy-tale-like voice, others are grounded in a more realistic approach (Tamaro, Viganò, Duranti, Sanvitale): but all of them share a stylistic peculiarity that I would label as "irony." Irony seems to work especially well when administered by women. (Bertone 79)

Siamo inscritte nel postmoderno, nella crisi di valori, del sociale, del politico in Italia anche del partitico — dove la scrittura può essere uno strumento per mettersi in rapporto (o negare il rapporto) con il mondo. Tale mondo - "cinico" e "asettico" per la Petri - entra a far parte della scrittura.

Il dibattito sul postmoderno in Nord America è tuttora molto vivace (molto è stato scritto anche sul rapporto tra postmoderno e femminismo), ${ }^{2}$ mentre in Italia, dopo anni di condanna del postmoderno come debole e deleterio da gran parte dei critici letterari, ultimamente sembra ospitare qualche voce alternativa. Alcuni esponenti del Gruppo '93 hanno etichettato il loro lavoro come "postmoderno critico": con la coscienza di una improponibile ed impraticabile avanguardia si opta per una riflessione e ricerca critica sulla produzione letteraria oggi. Scrive Biagio Cepollaro:

la querelle moderno-postmoderna è già tutta metabolizzata dal concreto fare testuale: la cultura postmoderna non è solo ideologia (e dunque, niente neomoderno) ma è anche espressione sintomatica di una condizione entro la quale tutti viviamo (tecnologie, dimensioni antropologiche della percezione, modi di comunicare, modi e atteggiamenti del mercato nel suo rapporto con i consumatori etc.). Le pratiche della citazione, il trattamento specifico dei materiali linguistici tendono ad allegorizzare un tipo di diagnosi complessa e non manichea, un tipo di risposta, in positivo all'“empasse" attuale. (Cepollaro 37)

Postmoderno e femminismo possono incontrarsi; secondo Linda Hutcheon, infatti:

It is not just a matter of feminisms having had a major impact on postmodernism, but perhaps postmodern strategies can be developed by feminist artists to deconstructive ends - that is, in order to begin the move towards change (a move that is not, in itself, part of the postmodern). (Hutcheon 149)

E ciò che si verifica nella narrativa della Petri: strategie postmoderne hanno funzione decostruttiva per cui non esiterei a parlare di postfemminismo postmoderno critico. La Petri rappresenta un quotidiano con situazioni assurde e grottesche in cui il personaggio-donna non è mai l'ombra, la debolezza, la passività, eppure non c'è inversione di ruoli o sopraffazione; si gioca, invece, ironicamente con il mondo maschile - dal lavoro al linguaggio, alla sfera sessuale. La rappresentazione del maschio in crisi, alla ricerca di un'identità e di cose da dire si può leggere come un'allegoria dell'attuale crisi della società. La Petri non aderisce al femminismo impegnato e militante; come molte della sua generazione che hanno vissuto il '77, il femminismo l'ha ingurgitato, digerito e ne ha preso le distanze. Io credo che lei parta dall'assunto che dell'uomo-donna non si debba parlare in 
termini di opposizione/contrapposizione ma che si debba, allo stesso tempo, rifiutare il femminile come "dispersione" come viene inteso nel discorso decostruzionista (si veda a proposito la risposta di Braidotti, Cavarero, De Lauretis all'indecidibilità della/sulla donna). ${ }^{3}$ All'interno di tale dibattito Patrizia Magli si chiede come essere donne "forti" sebbene inscritte nel discorso "debolista"; questo il suo progetto:

Non solo si tratta di una ridefinizione in grado di presentare la differenza come positiva, bensì di prospettare una differenza nel senso di pluralità di differenze. Una sorta di unità non monolitica, abitata dalla polifonia, polisessualità, nomadismo e molteplicità dell'essere. (Magli 19)

Questo discorso teorico diventa proficuo nell'analisi del lavoro della Petri in quanto, sebbene non si scriva esclusivamente di donne e per donne, bersaglio sono gli stereotipi della nostra società sessista; il maschio non è l'antagonista ma la vittima di tale società. A proposito farò riferimento a due racconti: "Il gambero blu" e "Nella grande città capitale". Così inizia "Il gambero blu":

Si alzò dal letto e corse in bagno.

Diamine, qui manca qualcosa! Si calò i pantaloni del pigiama, si tastò, lo cercò, cercò meglio e più volte, ma non c'era proprio più.

Corse di nuovo, questa volta verso il letto, alzò il lenzuolo per vedere se fosse rimasto lì. Nemmeno. Rifece il tragitto tra il letto e il bagno guardando scrupolosamente in terra. Niente, si era perso l'arnese. $(6)^{4}$

Dalla situazione e dal linguaggio questo racconto si presenta come un'assurda tragicommedia: tratta della frequente scomparsa e riapparizione dell'"arnese" del protagonista. Questi è un dirigente d'azienda - dunque ricco e borghese possiede un'auto sportiva e le donne che lo circondano - segretarie, dattilografe e telefoniste, oltre alle mogli dei colleghi - sono tutte sue attuali o ex amanti. E ossessionato dal sesso.

La voce narrante si adatta e si abbassa al linguaggio del protagonista, un linguaggio volgare e sessista. Chi lo contesta e mette in discussione non è una donna né la voce narrante bensì il suo arnese che sparisce ogni tanto in segno di protesta contro la noia terribile di una vita fatta solo di donne e minaccia di andarsene se non gli sarà concessa l'esperienza di un uomo. Nonostante sia disperato in assenza dell'arnese - tanto che si reca all'Ufficio oggetti smarriti e dai Carabinieri - il protagonista non ce la fa ad accettare l'idea di una relazione omosessuale; neanche ci prova, anzi. E così l'arnese scomparirà definitivamente perché annegato e lui inizierà una relazione promettente con una barista descritta come

scura di pelle, un po' avvizzita ma forte, vagamente mascolina nei gesti aspri e nell' atteggiamento. ("Il gambero blu" 105) [Corsivo mio] 
Non si parla di presa di coscienza, crescita e liberazione di donne ma questo racconto - parodia del sistema di valori del Maschio - vale più di un comizio. In romanzi femministi, in genere, l'emancipazione avviene grazie alla riscoperta e riappropriazione del corpo femminile, qui è l'organo sessuale maschile a prendere coscienza - in ottica femminista - e a dire no alla prevaricazione maschile. La parte omosessuale del protagonista si ribella, minaccia e si dissocia. Come dicevo prima, il problema non si pone in termini di opposizione uomo/donna e/o sesso maschile/femminile ma nella scoperta e rivalutazione delle differenze sessuali anche all'interno del singolo individuo. Come scrive Mario Mieli:

L'ambiente in cui viviamo ... è eterosessuale: in quanto tale costringe il bambino, colpevolizzandolo, a rinunciare alla soddisfazione dei propri desideri auto- e omoerotici e lo obbliga a identificarsi con un modello monosessuale di tipo eterosessuale mutilato. Ma non sempre ci riesce, evidentemente. (Mieli 7)

Secondo Mieli siamo tutti transessuali dato che in ognuno di noi è presente l'Altro sesso a livello cosciente o latente; nel protagonista del "Gambero blu" l'altro sesso è stato del tutto represso e il corpo si ribella: la natura si ribella alla cultura. Torna qui utile un termine molto esplicativo coniato da Mieli: "educastrazione", ovvero

l'influenza sull'individuo della società, del mondo "esterno", in cui vige una Norma monosessuale, che la repressione tramanda di generazione in generazione. (Mieli 13)

Che per la Petri la liberazione sessuale si realizzi con la liberazione della transessualità trova conferma in un altro racconto dal titolo "Nella grande città capitale". Il protagonista non ha nome; è genericamente, allo stesso tempo "specificamente", un uomo. Il giorno precedente il suo trentacinquesimo compleanno un uomo "non bello" ma "gradevole," per la prima volta in vita sua, nota che sta invecchiando:

... quel giorno per la prima volta cominciava a sentirsi diverso, e di questa diversità non sapeva ancora se rallegrarsi o preoccuparsi. Passeggiando per le vie del centro che si addensavano in colori imprevisti, sentì la necessità improvvisa di entrare in un negozio di biancheria femminile in via Borgognona e comperarsi un foulard. ("Nella grande città capitale" 10)

Subito dopo compra un rossetto ed un profumo da abbinare al foulard e, per la prima volta, si sente attratto dalle "vetrine extralusso per signore". E mentre avvengono questi cambiamenti, così vede la gente che osserva in un bar: "ognuno con il suo sesso ben determinato". Nel bagno del bar il colpo di scena: dopo una maschera facciale si ritrova donna e si passa dalla descrizione di lui a quella di lei:

Non si stupì minimamente quando guardandosi allo specchio vide il volto di una giovane e bellissima donna ... era donna, interamente donna nel volto e nel corpo, 
bella quasi eccessivamente, giovane un'altra volta e nuda. ("Nella grande città capitale" 15)

Adorna questo bellissimo corpo nudo con il foulard, il profumo ed il rossetto; lascia nel bagno organo sessuale ed abiti maschili e, incurante della sua nudità e fiera del suo corpo, si avvia. Tutti gli sguardi sono per lei e d'ora in poi vivrà "incalcolabili storie d'amore e di erotismo".

In questo racconto alla percezione transessuale che genera una prima confusione segue la liberazione transessuale che si realizza con la coscienza del proprio corpo o meglio, dei segni del corpo; ad esempio, leggiamo:

La sua camminata oltre alla velocità aveva acquistato anche un non so che di morbido, di più elastico; e le mani, che teneva lungo le cosce, col movimento del corpo ad ogni passo lo toccavano procurandogli una strana e piacevolissima sensazione. ("Nella grande città capitale"13)

Ad un corpo maschile, vecchio, stanco e senza sensazioni succede un bellissimo corpo nudo di donna che vibra e vive. Il foulard, il rossetto ed il profumo - in quanto ornamenti e accessori femminili — hanno la funzione di sottolineare l'erotismo femminile. Perniola parla dell'erotismo come relazione tra veste e nudo nelle arti figurative dove l'erotismo si dà nel "transito" dall'uno all'altro stato:

L'erotismo si manifesta nelle arti figurative come relazione tra vestito e nudo. La sua condizione è dunque la possibilità di un movimento, di un transito dall'uno all'altro: se ad uno dei due termini viene attribuito un significato primario ed essenziale a discapito dell'altro, manca la possibilità stessa del transito e quindi dell'erotismo. In tal caso alla veste o al nudo è assegnata una dimensione assoluta. $(85)^{5}$

Allo stesso modo, il foulard della Petri non veste, non nasconde il corpo nudo ma gioca con esso a coprire/scoprire a simboleggiare la felice metamorfosi. ${ }^{6}$

Petri è molto attuale con questo racconto dato che in Italia si parla molto oggi - anche con preoccupazione - del travestimento transessuale come fenomeno imponente. Ha ragione Vattimo quando afferma che il travestimento transessuale non è dovuto all'affermarsi di una cultura omosessuale ma ad una insofferenza per l'identità forte, anche sessuale; scrive:

... anche la riflessione filosofica ha cominciato a collocare il tema dell'alterità, cioè del rispetto e dell'ospitalità verso 1'altro, all'interno stesso del singolo: solo se uno riconosce la pluralità (anche di ruoli sessuali che porta in sé) può sperare di cominciare a capire qualcosa degli altri. (46-47)

Ho fatto riferimento a due racconti che hanno specificamente a che fare con la sfera sessuale, ma le coordinate maschile/femminile sono una costante nei racconti della Petri. ${ }^{7}$ Volgiamo la nostra attenzione al romanzo $I l$ ritratto del disarmo: il protagonista, Pillo, è uno scrittore che lotta, giorno dopo giorno, per trovare ispirazione alla scrittura, ma inutilmente. Si ritrova a riscrivere sempre la stessa storia mentre le altre due protagoniste, Marsilia - l'amica scrittrice che scrive e 
pubblica - e Oliviera - la moglie dinamica ed indipendente, sono eroine positive. Anche qui si gioca con le categorie maschile/femminile: Oliviero e Marsilio, eroi della Chanson de Roland, diventano qui due eroine; in proposito la Petri commenta:

Io non ho voluto dare un aspetto spiccatamente femminile alle due donne. Marsilia specialmente è asessuata. M'ha ispirato la Chanson de Roland, come stanno a dimostrare i nomi cambiati al femminile: Marsilio capo dei pagani e Oliviero, l'amico di Rolando. In quell'opera, un uomo che sta per morire, con una frase ambigua, forse bisex, può mormorare teneramente all'amico: "Io ti amo". (Gerardis)

Orlando/Pillo, unico maschio/protagonista, combatte una guerra che perderà. Egli è un fallito nella vita e nella scrittura:

A chi sosteneva che la vita o la si vive o la si scrive, Petri sembra opporre il ruvido scontro con il quotidiano, da cui solo può nascere una scrittura autentica, come dimostra il caso di Marsilia, che riuscirà invece a pubblicare il suo libro. (Brevini $18-20)$

Pillo è inscritto nel suo piccolo mondo quotidiano in cui non c'è spazio per passionalità e dove la comunicazione con l'esterno è ridotta al minimo. Le donne-artiste nella narrativa di Petri non hanno problemi a creare arte mentre Pillo è costretto a deporre le armi del disarmo:

Altri fogli bianchi sulla scrivania che cercava di riempire con parole che gli sembravano sempre poco inventate, quasi un'imitazione delle sue letture che non sapeva come frenare, ogni volta tradito da una buona fede che durava solo il tempo della scrittura. (Il ritratto del disarmo 12)

Un altro esempio di impotenza a scrivere la ritroviamo nel racconto "Bloom's Day 1978". Il protagonista è un giornalista a cui davanti alla macchina da scrivere gli si chiude il cervello e solo con una donna al suo fianco riesce a scrivere:

... quando ho una compagna accanto ci riesco, poi quando rimango solo convinto di farcela ... ripiombo in questo inferno 'nfernale. ("Bloom's Day 1978" 61)

La protagonista/scrittrice di "Un falco tra l'alba e il tramonto," invece, scrive con fatica ma scrive. $E$ il racconto è una riflessione sul farsi della storia; leggiamo:

E io sto qui a scrivere due righe che buttarle giù è una gran fatica, tanto che poi mi alzo e me ne vado in bagno per spezzare. ("Un falco tra l'alba e il tramonto" 129)

Mentre lei cerca di inventare storie, il marito, passivo e taciturno, si lascia risucchiare dalle storie della televisione. Qui la creatività, la capacità di inventio diventa una sfida contro le storie propinate dalla TV: 
Non ci ho mai avuto simpatia per quell'aggeggio accendi e spegni mancante sia di discrezione che di tatto, dove chi l'ha uccisa si crea l'alibi e tu giù a bertela tutta fino alla fine della trasmissione, tutto in congetture e lambiccamenti di cervello a fregartene di tutto quello che hai fatto durante il giorno che pare quasi che debba entrarti in casa e prenderti a colpi di accetta se non si costituisce lui o l'acciuffano loro. ("Un falco tra l'alba e il tramonto" 136)

Se è vero che la società postmoderna è la società dei mass media, è anche vero che il tentativo di sfuggire al linguaggio e alle storie dei media è un atteggiamento postmoderno critico. Postmoderno critico a livello contenutistico ma anche a livello linguistico e stilistico. Si potrebbe anzi parlare di un nuovo sperimentalismo e indubbiamente Giorgio Manganelli rappresenta un modello; ${ }^{8}$ al linguaggio banale e ripetitivo dei mass media, la Petri, come Manganelli - con atteggiamento neobarocco - contrappone un linguaggio colto, nuovo e ironico. Dice Manganelli:

... l'italiano si sta impoverendo in maniera paurosa. Sta prevalendo una corrente che inquina la nostra letteratura, la corrente diciamo così del basic italian, per cui si scrivono romanzi di inaudita lunghezza in basic italian, dove il lessico finale risulta un lessico alla portata di chiunque abbia studiato alla Berlitz per un corso di cameriere. (Drudi Demby 47)

La trasgressione e distorsione linguistica mina il basic italian a cui siamo esposti; così, sulla pagina della Petri, ad esempio, all'ora di punta segue l'odore di punta ("Colazione sull'erba" 24); è frequente l'uso delle maiuscole: "OH BIMBA!!! TE TU M'HAI DATO L'AZZURRO!!" ("Bloom's Day 1978" 44); a volte le parole vengono fuse insieme (ad esempio: "passionalestupratoreaddestratore") e altre volte troncate (ad esempio: "sta-va pro-prio be-ne e c'e-ra pu-re l'aria di pri-ma-ve-ra"). Si gioca con il linguaggio sinistrese (in particolare in "Bloom's Day 1978"), con parole straniere: “. . . quella grande America era 'more' anche nel clima" ("Il gambero blu" 99) e con interferenze dialettali o straniere:

la moglie americana mi salutava le prostitute per strada "ciauu", cazzo le conosci? sono amiche tue? "zi, fecciamou zempre la zpesa inziemi tutti li matinei". ("Bloom's Day 1978" 45)

Le parole, per la Petri come per Manganelli, sono soprattutto suono e vanno toccate e ascoltate oltre che scritte e lette. Un esempio di sonorità da Manganelli:

Io amo $\mathrm{i}$ libri boccone, che stanno in una mano come un tiepido marrone, che promettono un cibo leggero e dilettoso. (Manganelli, Discorso dell'ombra e dello stemma 84)

ed un esempio dalla Petri: 
Perchè a lui la mogliettina piace canterina dalla sera alla mattina. Cinguetto in cucina .... Cinguetto passerina. ("Un falco tra l'alba e il tramonto" 131)

Lo sperimentalismo si esprime a vari livelli. I confini tra i generi letterari non sono netti; troviamo, ad esempio, commenti a mo' di didascalie teatrali, si aprono lunghissime digressioni - a volte di più pagine - e un periodo spesso copre quasi lo spazio di una pagina. A volte volutamente si crea ambiguità tra la voce narrante e il discorso diretto e indiretto poiché si fa scarso uso delle virgolette e altri segni indicatori del discorso diretto:

E poi raccontava, raccontava tutto di tutte le sere e diceva Sapessi quella sera! alzando le braccia verso l'alto . . . Dice: Facciamo un caffè e se ne va in cucina col passo scalzo e umido.... (Il ritratto del disarmo 190-91)

Spesso si crea ambiguità tra il soggetto e l'oggetto o tra diversi soggetti come nel brano che segue:

Entrava in una rosticceria per comprare della pizza a taglio. Proprio all'entrata c'era un cane da caccia bianco e marrone; non era stata ancora sfornata e non aveva tempo da perdere, muoveva la coda col muso appoggiato alla porta . . . (Il ritratto del disarmo 59)

All'assenza di categorie sessuali rigide si affianca l'assenza di categorie narrative rigide. Si "transita" dal genere femminile al maschile, dal narrativo al teatrale, dall'italiano all'inglese, dal discorso diretto all'indiretto, da un enunciatore ad un altro e la voce narrante camaleonticamente, alla Zelig, si adatta. Sono tutte caratteristiche queste che hanno funzione di straniamento e che possiamo definire postmoderne, neobarocche o neosperimentali. Dello stile del romanzo della Petri, Pedullà scrive:

Nel romanzo sminuzza la narrazione in centinaia di frammenti di poche righe o, al massimo di due pagine. Frantumi caricati di elettricità o comunque di elevata temperatura, secondo una strategia espressionista? Invece no, siamo prossimi al grado zero, sul quale fa freddo. C'è però tensione, che si nota quando viene spezzata. (Pedullà)

E che lo sperimentalismo linguistico e stilistico sia l'essenza della narrativa della Petri ce lo dice lei stessa:

Il mio romanzo: duecento pagine di gelo in cui l'unico calore viene dal linguaggio. (Rusconi 99) 


\section{NOTE}

1 La Petri continua a scrivere e a pubblicare racconti; si veda il racconto "Roma sparita" nell'antologia del '93 Racconta 2.

2 Si vedano Flax, Hutcheon, Lee, Lovibond, Nicholson, Owens, Sherzer, Waugh: in ambito italianista si veda Holub.

3 Si vedano AA.VV. Diotima. Il pensiero della differenza sessuale, AA.VV. Diotima. Mettere al mondo il mondo, Braidotti, Cavarero, De Lauretis, Jardine e Smith, Magli, Marcuzzo e Rossi-Doria, Morino.

4 Di questo racconto Manganelli dice: "Il racconto che dà il titolo al libro è uno dei racconti più belli che abbia letto in questi anni, di una genialità costruttiva straordinaria: un attacco gogoliano, divagazioni audaci quanto controllate, ed un inserto - un soggiomo americano - che è un autentico pezzo di bravura, un gioco di colori e immagini tra il grottesco e il sinistro..." (Manganelli, "Fra poesia e licantropia").

5 Per la funzione del velo nelle arti figurative, si veda, nello stesso volume, anche il paragrafo "L'erotica dello spogliare: nudo e velo" (90-94).

6 Secondo Amoroso, lo scopo della Petri sembra essere "quello di cogliere la sparente coscienza, di individuare il costituirsi di una nuova identità, priva di memorie, legami, e coinvolta festosamente nel fatale compiersi di una metamorfosi".

7 Altri testi da affiancare a quelli della Petri per stimolanti raffronti sul "transito" dal maschile al femminile potrebbero essere Orlando di Virginia Woolf, The Passion of New Eve di Angela Carter, The Female Man di Joanna Russ.

8 Lei stessa invita Pizzuto, in un'intervista, ad un confronto: "Ed è qui che Romana Petri mi invita ad una lettura comparata: quella di un suo racconto impalpabile, austero, tristissimo ('Nel diverso percorso') con alcune pagine conclusive di Amore, opera carismatica di Giorgio Manganelli. 'lo credo — afferma la Petri — che la tensione metafisica e la segreta teatralità di quel che accade ai personaggi sia innegabile. Spero solo di essere una degna 'allieva"" (Pizzuto). In una conversazione telefonica (14 ottobre 1992), la Petri alla mia domanda sul rapporto con Manganelli ha risposto di considerarsi "una sua creatura".

\section{OPERE CITATE}

AA.VV. Diotima. Il pensiero della differenza sessuale. Milano: La Tartaruga, 1987.

AA.VV. Diotima. Mettere al mondo il mondo. Milano: La Tartaruga, 1990.

Amoroso, Giuseppe. "L'angoscia penetra anche nel bizzarro". La Gazzetta del Sud (21 agosto 1990).

Bertone, Manuela. “A Conversation with Dacia Maraini”. Harvard Review (Spring 1992): 76-79.

Braidotti, Rosi. Patterns of Dissonance. New York-London: Routledge, 1991.

Brevini, Franco. "Il male di scrivere". Panorama (29 settembre 1991): 18-20.

Carter, Angela. The Passion of New Eve. London: Virago Press, 1982.

Cavarero, Adriana Nonostante Platone. Roma: Editori Riuniti, 1991.

Cepollaro, Biagio. "La compresenza conflittuale. Quattro equivoci sintomatici sulle vicende del gruppo '93". Baldus I (agosto 1991): 37-40.

De Lauretis, Teresa. Technologies of Gender. Bloomington-Indianapolis: Indiana UP, 1987.

Drudi Demby, Lucia. "Giorgio Manganelli". Il caffé (giugno 1965): 42-48. [Conversazione con Giorgio Manganelli.]

Flax, Jane. Thinking Fragments. Berkeley: U of California P, 1990.

Gerardis, Adriana. "Pillo, con la missione di scrivere". La Gazzetta del Sud (25 luglio 1991). [Intervista a Romana Petri.]

Holub, Renate. "Weak Thought and Strong Ethics: The 'Postmodern' and Feminist Theory in Italy". Annali d'Italianistica 9 (1991):124-43. 
Hutcheon, Linda. "Postmodernism and Feminisms". The Politics of Postmodernism. New YorkLondon: Routledge, 1989. 141-68.

Jardine, Alice e Paul Smith, eds. Men in Feminism. New York-London: Methuen, 1987.

Lee, Janet. "Care to Join me in an Upwardly Mobile Tango?" The Female Gaze. Eds. Lorraine

Gamman e Margaret Marshment. Seattle: The Real Comet Press, 1989. 166-72.

Lovibond, Sabina. "Feminism and Postmodernism". New Left Review 178 (1989): 5-28.

Magli, Patrizia. "ll segno della differenza". Le donne e i segni. Ed. Patrizia Magli. Ancona: Transeuropa, 1988. 11-22.

Manganelli, Giorgio. Discorso dell' ombra e dello stemma. Milano: Rizzoli, 1982. . "Fra poesia e licantropia". Il Messaggero (26 marzo 1990).

Marcuzzo, Maria Cristina e Anna Rossi-Doria, eds. La ricerca delle donne. Studi femministi in Italia. Torino: Rosenberg e Sellier, 1987.

Mieli, Mario. Elementi di critica omosessuale. Torino: Einaudi, 1977.

Morino, Alba. "L'androgino. Femminile e maschile nella scrittura e nella lettura". Scritture, scrittrici. Ed. Maria Rosa Cutrufelli. Milano: Coop-Longanesi, 1988. 85-93.

Nicholson, Linda, ed. Feminism/Postmodernism. New York-London: Routledge, 1990.

Owens, Craig. "The Discourse of Others: Feminists and Postmodernism". Postmodern Culture. Ed.

Hal Foster. London: Pluto Press, 1983. 57-82.

Pedullà, Walter. "Ulisse è stanco, ha fatto il sessantotto". Il Messaggero (15 giugno 1991).

Pemiola, Mario. Transiti. Come si va dallo stesso allo stesso. Bologna: Cappelli, 1985.

Petri, Romana. ll baleniere delle montagne. Milano: Rizzoli, 1993.

. "Colazione sull'erba". Il gambero blu. 19-27.

. "Un falco tra l'alba e il tramonto". Il gambero blu. 127-39.

. "Il gambero blu". Il gambero blu. 63-107.

.ll gambero blu e altri racconti. Milano: Rizzoli, 1990.

. "Nella grande città capitale". Il gambero blu. 7-17.

.ll ritratto del disarmo. Milano: Rizzoli, 1991.

. "Roma sparita". Racconta 2. Eds. Rosaria Guacci e Bruna Miorelli. Milano: La

Tartaruga, 1993. 223-36.

."Bloom's Day 1978". Il gambero blu. 29-62.

Pizzuto, Angelo. "L'ordinario quotidiano sconfina nel fantastico". La Sicilia (15 febbraio 1990).

[Intervista a Romana Petri.]

Rusconi, Marisa. "Le neo-romanze". L'Espresso (24 febbraio 1991): 94-99.

Russ, Joanna. The Female Man. Boston: Beacon Press, 1986.

Sherzer, Dina. "Postmodernism and Feminisms". Postmodernism and Contemporary Fiction. Ed.

Edmund J. Smyth. London: B.T. Batsford Ltd, 1991. 156-68.

Vattimo, Gianni. "Con la signora Coriandoli contro I'intolleranza". L'Espresso (22 marzo 1992): 46-47.

Waugh, Patricia. Feminine Fictions. Revisiting the Postmodern. New York-London: Routledge, 1989.

Woolf, Virginia. Orlando. San Diego-New York-London: Harcourt Brace, 1993. [1928.] 